\title{
Surgical management and follow-up of lateral skull base tumors: An 8-year review
}

\author{
JIE KONG ${ }^{1,2}$, HONG-YU YANG ${ }^{1}$, YU-FAN WANG ${ }^{1}$, HUI-JUN YANG ${ }^{1}$, SHI-YUE SHEN ${ }^{1}$ and FENG WANG ${ }^{1}$ \\ ${ }^{1}$ Department of Oral and Maxillofacial Surgery, Peking University Shenzhen Hospital, Shenzhen, Guangdong 518036; \\ ${ }^{2}$ Peking University Shenzhen Hospital Clinical College, Anhui Medical University, Hefei, Anhui 230032, P.R. China
}

Received October 15, 2016; Accepted December 8, 2016

DOI: $10.3892 / \mathrm{mco} .2017 .1126$

\begin{abstract}
The purpose of the present article was to describe the diagnostic evaluation of, and surgical approaches to, lateral skull base tumors (LSBTs). The study is a retrospective review of 21 patients diagnosed with tumors that involve lateral skull base ( 8 with malignant diseases and 13 with benign lesions) who were surgically treated during a 8 -year period. The transparotid-transmandibular (38\%) was the most commonly performed surgical procedure, followed by the transmandibular (24\%), the transmaxillary $(24 \%)$, the transcervical approach $(10 \%)$ and the combined approach $(4 \%)$. The surgical procedures were uneventful and there were no postoperative mortalities. Complications were encountered in 12 cases, and morbidity was not remarkable during the perioperative stages. After an average follow-up of 46 months, only 1 of 14 patients with benign diseases had a recurrence following the resection of a pleomorphic adenoma. Of 7 patients with malignant tumors, 5 are alive with no evidence of disease. The majority of the benign lateral skull base tumors can be removed surgically with a low rate of complications and recurrence. However, malignant neoplasms carry a poor prognosis and a low rate of disease-free survival.
\end{abstract}

\section{Introduction}

Skull base surgery is a new cross edge discipline which has been formed in recent years and primary skull base tumor has less incidence in clinical relatively. Most of the skull base tumors treated by oral and maxillofacial surgery mainly involve the lateral skull base (LSB). The anatomy of this area had been described in detail: This area is a well-concealed and complex anatomical region with significant functions and narrow surgery view (1-3). All of these account for the diversity of surgical approaches for removal of lateral skull base

Correspondence to: Professor Hong-Yu Yang, Department of Oral and Maxillofacial Surgery, Peking University Shenzhen Hospital, 1120 Lian Hua Road, Shenzhen, Guangdong 518036, P.R. China E-mail: hyyang192@hotmail.com

Key words: tumor, lateral skull base, surgical management tumors (LSBTs). Most LSBTs are benign (65-75\%), and they usually originate from the salivary glands comprising 40-50\% of the total. The rest are neurogenic $(20 \%)$ or otherwise (20\%) (3-7). Manifestations of LSBTs include a mass in the oropharynx or the upper neck, changes in voice, cranial nerve deficits, and so forth. However, in some cases, LSBTs may go undetected for a long time, and they usually present as an asymptomatic mass $(6,8,9)$. A variety of surgical approaches have been described for management of LSBTs, the most common among them including the transmandibular, the transmaxillary, transparotid-transcervical and the transcervical approaches. The decisive factor that affects the option of the surgical approach is which one will maximize exposure for intact tumor excision while minimizing functional and aesthetic deficits. This article describes our 8-year experience of managing these tumors, mainly concerning which current diagnostic evaluation is used for the determination of the operational plan, and our surgical approaches for the removal of LSBTs.

\section{Patients and methods}

Patient population. Between January 2007 and August 2015, 21 patients ( 13 male and 8 female) ranging in age from $25-70$ years (mean, 46 years) underwent operations for lesions involving the LSB. Patients were evaluated by an oral maxillofacial surgeon and all were operated on by the same surgical team. Patients were followed for an average of 36 months. The medical charts were analyzed retrospectively.

Clinical presentation. Patients mainly presented with local symptoms and signs. Maxillofacial pain and facial mass were the most common types experienced by the patients. Other manifestations, including facial paralysis, trismus, dysphonia, dysphagia, foreign body sensation, hoarseness, visual change, rhinocleisis, and so forth, can also be observed in certain cases. If lesions invade the cavernous sinus, palsies of partial cranial nerves may occur.

Imaging. Imaging modalities such as computer tomography (CT) and magnetic resonance imaging (MRI) are of great importance for determining the extent of the lesion, to depict its relationship with vital vessels, to rule out any intracranial involvement, to assess its resectability, and to guide the operating 
surgeon through planning the right surgical approach $(2,5)$. We make a comparison between a contrast computerized tomographic (CT) scan and a basic magnetic resonance imaging (MRI), and we consider that MRI with gadolinium is preferred to CT in terms of diagnosing LSBTs with the exception of its cost. In addition, angiography is recommended for all enhancing lesions or vascularized masses, particularly if imaging shows a widening of the carotid bifurcation. Visualization of a vascular flow void on a CT or MRI study is usually adequate, but computerized tomographic angiography (CTA) or magnetic resonance angiography (MRA) may occasisionally be added for supportive evidence for vascular tumors. Moreover, 3D CT or 3D MRI reconstruction makes it convenient to observe neoplasm invasion by multiplanar graphics. So broadly speaking, high-resolution images are always essential for LSBTs (Fig. 1A-E).

Surgical techniques. Completing surgical excision and minimizing morbidity are the main purposes of treatment of LSBTs. However, patients should be well informed about the potential for complications during the perioperation. Varieties of surgical approaches for the resection of LSBTs were used: The transmandibular, transmaxillary, transparotid-transmandibular, transcervical and the combined approaches. All approaches have their own specifications, advantages and disadvantages. The most common criterion for choosing an appropriate approach is maximizing the exposure for completing tumor excisions and the conservation of anatomic structures, while minimizing the functional and esthetic defects of patients. In addition, the plastic reconstruction, which consists of repair of the craniofacial tissue defects, osteosynthesis, and elimination of the operative cavity, is required for the patients.

The transmandibular approach. The transmandibular approach is adopted in cases where patients suffer from extremely large tumors, especially tumors with vascular lesions or tumors with lesions invading the skull base. They need better exposure to allow a safe excision. A cutaneous incision extending from the mastoid to the submandibular region in the midline is performed. For fear of damage to the marginal mandibular branch of the facial nerve, we identify $1.0-1.5 \mathrm{~cm}$ margins anteriorly and inferiorly to the mandibular angle to protect this nerve. Once the mandible is split, we call it 'mandibulotomy'. In the case of mandibulotomy, nasotracheal intubation is used in order to permit evaluation of the occlusion. Through this method, surgeons can get a better view. Moreover, prior to completing the osteotomy, using two titanium miniplates is performed for pre-plating. This method allows surgeons to open the superior portion of the osteotomy without completely separating the inferior bone, thus holding the condylar position and removing the tumor under direct visualization of the surrounding tissues. Finally, the mandible is repositioned to the initial position after tumor resection.

The transmaxillary approach. The transmaxillary approach is made by a Weber-Ferguson incision, and a fronto-temporal incision is marked for preparation if the transmaxillary approach alone is not sufficient to have the adequate operation field. A cheek flap, including half of the upper lip, is turned laterally to expose the anterior portion of the maxilla. Osteotomy of the maxilla comprises inferior and lateral orbital walls, malar process, maxillary process and palatal suture. Afterwards, resection of the partial orbital floor and lateral wall is performed; thereby surgeons can gain a wide access to the lesion region. If necessary, the posterior maxillary and the pterygoid plate are removed to create more posterior space. When the lesion excision is completed, the relatively large operative cavity is packed with the vascularized flap, although secondary plastic reconstruction may still be required.

The transparotid-transmandibular approach. In the transparotid-transmandibular approach, a Blair incision is performed in the preauricular skin, made around the earlobe and then extended to the neck. Usually, this approach is suitable for LSBTs that are derived from the deep lobe of the parotid gland. A parotidectomy is performed after identification of the facial nerve and all of its branches. Tumors of the deep lobe of the parotid gland often stay underneath the branches of the facial nerve. In such cases, the branches of the facial nerve are separated from the capsule of the tumor. Then, the tumor is taken out from this space with a combination of blunt and sharp separation under direct visibility. Furthermore, a transparotid-transcervical approach can be performed through an extended incision.

The transcervical approach. In the transcervical approach, surgeons will make a curved transverse skin incision at $2-3 \mathrm{~cm}$ underneath the lower border of the mandible. This approach is indicated for a minority of the dissection of LSBTs that originate from the inferior portion of the parapharyngeal space. However, through the transcervical approach alone, it is always difficult to obtain enough visibility and to remove LSBTs completely. Therefore, surgeons will consider adopting a transparotide-transcervical approach in order to increase exposure and facilitate tumor removal.

The combined approach. The combined approaches can be classified as the transcervical-transoral, transparotid-transcervical,transmandibular-transcervical,transmastoid-transcervical, transmaxillary-transparotid-transmandibular approaches (Fig. 1F, I), etc. In certain complex cases, surgeons may find it hard to perform a tumor resection with one single approach. Accordingly, single approaches are modified into these combined approaches for complete mass removal. The real difficulty of the combined approaches is to finish preoperative surgical planning and to adjust intraoperative surgical planning. As a consequence, on account of the characteristics of multiplicity and individuation, the combined approaches require that surgeons must be very experienced and have excellent resourcefulness. Otherwise, the operations still will be have the desired effects.

Mandibulectomy. A mandibulotomy is necessary when large, recurrent or malignant tumors require better exposure to facilitate removal. On account of its postoperative complications (such as facial scar and malocclusion), an important consideration is to try to avoid dividing the mandible as far as possible. It is our opinion that this radical approach may serve an important role, where appropriate. 

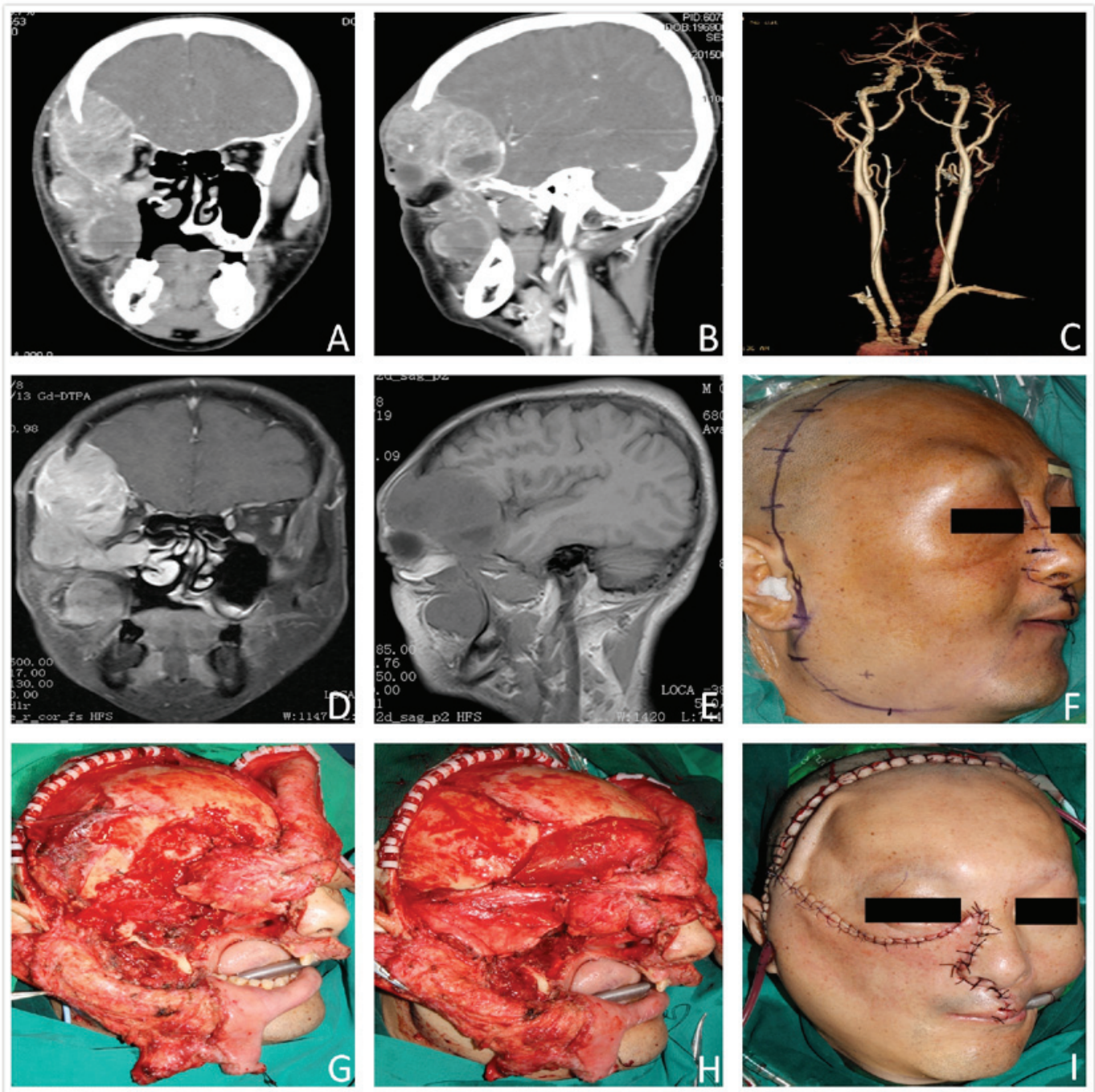

Figure 1. Typical case: The 43-year-old male underwent a modified combined approach to resect a maxillary sinus myxofibrosarcoma. Preoperative coronal (A), sagittal (B) CT scans showing a maxillary sinus mass involving the lateral skull base. Preoperative CTA (C) scan showing the relation of the major vessels of the operative area. Preoperative coronal T1-weighted (D) and sagittal T1-weighted (E) MRI scan showing a maxillary sinus mass involving the lateral skull base. Intraoperative photograph (F) showing intraoperative mark of the operation approach. Intraoperative photograph $(\mathrm{G})$ showing the removal of the mass and the adjacent tissues. Intraoperative photograph $(\mathrm{H})$ showing the plastic reconstruction of the tissue defects. Intraoperative photograph $(\mathrm{I})$ showing the suture of incision after resection and reconstruction.

The plastic reconstruction. When the tumor resection is finished, the dura defects are reconstructed with autogenous fascia or artificial materials (Fig. $1 \mathrm{G}$ and $\mathrm{H}$ ). Facial tissue defects are recovered with regional flaps or free flaps. Large mucosal oroparapharyngeal defects need a radial-forearm fasciocutaneous flap. Combined skin and mucosal defects require free flaps, which may be folded over themselves with the epithelialized surface inside. Craniofacial skeletons moved for exposure are replaced and stabilized with titanium plates and screws.

Postoperative measures. Patients, following the relevant surgery, should continue to be checked regularly. They should be re-examined every 3 months within 3 years, and then the rechecking schedule should be changed to every 6 months. If necessary, postoperative chemoradiotherapy may be performed 1 week after the operation, while postoperative radiotherapy may be carried out 1 week after taking out the suture.

\section{Results}

This was a retrospective analysis of LSBTs in 21 patients (13 males; 8 females) treated primarily by surgery, whose mean age was 46 years (range 25-70 years). Patients who had undergone surgery for LSBTs in other institutions and patients with other tumors were excluded from the study (Table I).

The incidence and frequency of symptoms were analyzed (Table II). The common symptoms were maxillofacial pain and dysphagia, which presented in 7 patients (33\%). Other, less frequent symptoms included facial paralysis (14\%), foreign body sensation (10\%), trismus (5\%) and dysphonia (5\%). From Table II, the most frequent clinical signs were facial mass (29\%) and parotid mass (24\%). It is noteworthy that the incidence of asymptomatic patients was significant. A total of $>50 \%$ of the patients (11 patients; $52 \%$ ) had an asymptomatic mass. It was usually identified during a conventional checkup, or accidentally detected after an imaging study for other diseases was performed. 
Table I. Clinical data.

\begin{tabular}{lcclllll}
\hline No. & Sex & Age (yrs) & $\begin{array}{l}\text { Benign/ } \\
\text { malignant }\end{array}$ & $\begin{array}{l}\text { Surgical } \\
\text { approach }\end{array}$ & Complication & $\begin{array}{l}\text { Follow-up } \\
\text { (months) }\end{array}$ & Outcome \\
\hline 1 & F & 33 & Benign & TMY & NC & 26 & NED \\
2 & F & 42 & Benign & TMR & TPOFN & 75 & NED \\
3 & M & 51 & Benign & TMY & TPOFN & 65 & NED \\
4 & M & 40 & Malignant & TPTM & NC & 61 & LRBT \\
5 & M & 25 & Benign & TMR & NC & 64 & NED \\
6 & F & 61 & Benign & TMR & TPOFN & 50 & NED \\
7 & F & 48 & Malignant & TPTM & NC & 32 & NED \\
8 & M & 70 & Benign & TPTM & TPOFN & 62 & NED \\
9 & M & 64 & Malignant & TPTM & NC & 43 & NED \\
10 & M & 46 & Benign & TMY & NC & 101 & NED \\
11 & F & 35 & Benign & TPTM & NC & 49 & NED \\
12 & F & 30 & Malignant & TC & UPOTVC & 14 & LRMT, DOD \\
13 & F & 62 & Benign & TPTM & Bleeding & 30 & NED \\
14 & F & 48 & Benign & TPTM & Frey's syndrome & 56 & NED \\
15 & M & 43 & Malignant & TMR & Dysphagia & 5 & NED \\
16 & M & 57 & Benign & TC & UPOTVC & 64 & NED \\
17 & M & 36 & Benign & TPTM & NC & 13 & NED \\
18 & M & 40 & Malignant & TMR & Trismus & 11 & NED \\
19 & M & 54 & Benign & TMY & NC & 45 & NED \\
20 & M & 33 & Benign & TMY & TPOFN & 78 & NED \\
21 & M & 55 & Malignant & Combined & UPOTVC & 19 & LRMT, DOD \\
\hline
\end{tabular}

TPTM, transparotid-transmandibular; TMR, transmandibular; TMY, transmaxillary; TC, transcervical; NC, no complication; TPOFN, temporary paralysis of facial nerve; UPOTVC, unilateral paralysis of the vocal cords; NED, no evidence of the disease; LRBT, local recurrent benign tumor; LRMT, local recurrent malignant tumor; DOD, dead of disease.

All patients underwent surgical operation of their tumors. The approach to the tumors is given in Table III. The most frequent operative technique was the transparotid-transmandibular approach in 8 cases (38\%). In 5 (24\%) patients, a transmandibular approach was used. A transmaxillary approach was applied in 5 (24\%) patients as well. The number of patients for whom a transcervical approach was applied was $2(10 \%)$. Finally, $1(5 \%)$ patient was subjected to a combined approach treatment. Moreover, mandibulotomy was required in $6(29 \%)$ cases.

Complications of our surgical treatments were noted in 12 patients (Table IV). Temporary paralysis of the facial nerve (mainly the marginal branch) was observed in 5 patients. Frey's syndrome was seen in 2 patients, but over a period of time, this gradually faded. Unilateral paralysis of the vocal cords was observed in 2 patients, followed by bleeding in 1 patient ( $24 \mathrm{~h}$ following surgery), dysphagia in 1 patient and trismus in 1 patient.

The results of histopathological analysis are presented in Table V. In our series, there is a distinct predominance of benign tumors [in 14 patients (67\%), compared with malignant tumors in 7 patients (33\%)]. The most frequent one was pleomorphic adenoma (9 patients) among the benign tumors. The rest of the benign tumors may be grouped under the heading 'sundry'. In addition, the histopathological distribution of the 7 malignant neoplasms was mixed. We found that there was no clear tendency about the types of malignant neoplasms in our series from Table V.

There were no identified cases of mortality in the perioperative period and the mean follow-up was 46 months. Regarding postoperative recidivation, of the $14(66.7 \%)$ patients with benign tumors, only 1 case of pleomorphic adenoma was recorded 5 years after the initial surgery. Beyond that, malignant tumors occupied $33.3 \%$ of the patients ( 7 patients), and the follow-up revealed 2 patients $(9.5 \%)$ died due to having a local recurrent malignancy. Postoperative radiotherapy was used in 5 patients, and 2 patients were treated with combined chemoradiotherapy. The patients with malignant tumors had ordered controls that included clinical examination, lung X-ray and CT (1 month after completing radiotherapy; if patients do receive postoperative chemoradiotherapy, they should have a CT scan after finishing every third cycle of the chemotherapy). In this group of patients, 3 were disease-free, 2 were alive although they still had the disease, and 2 died of recurrence or metastasis in the follow-up period (14 and 19 months following surgery).

\section{Discussion}

The LSB is the lateral part of the skull base that includes the deep areas of the temporal and zygomatic bone. There are many vital structures in this area, which contains the parotid 
Table II. Clinical features of LSBTs.

\begin{tabular}{lrr}
\hline & & Total \\
\cline { 2 - 3 } & $\mathrm{N}$ & $\%$ \\
\hline Symptom & & \\
Maxillofacial pain & 7 & 33 \\
Dysphagia & 7 & 33 \\
Facial paralysis & 3 & 14 \\
Foreign body sensation & 2 & 10 \\
Trismus & 1 & 5 \\
Dysphonia & 1 & 0 \\
Hoarseness & 0 & 0 \\
Visual change & 0 & 0 \\
Rhinocleisis & 0 & 5 \\
Sign & & 52 \\
Facial mass & 6 & 24 \\
Parotid mass & 6 & 10 \\
Oropharyngeal mass & 5 & 5 \\
Neck mass & 2 & \\
No symptoms or signs & 1 & \\
\hline
\end{tabular}

LSBT, lateral skull base tumor.

Table III. Surgical approach to LSBTs.

\begin{tabular}{lrr}
\hline & \multicolumn{2}{c}{ Total } \\
\cline { 2 - 3 } Surgical approach & $\mathrm{N}$ & $\%$ \\
\hline 1. Transparotid-transmandibular & 8 & 38 \\
2. Transmandibular & 5 & 24 \\
3. Transmaxillary & 5 & 24 \\
4. Transcervical & 2 & 10 \\
5. Combined & 1 & 4 \\
Total & 21 & 100 \\
\hline
\end{tabular}

LSBT, lateral skull base tumor.

gland and minor salivary glands, the VII, IX, X and XI cranial nerves, the cervical sympathetic nerve, lymph nodes, the internal jugular vein, the external carotid artery and its branches, and especially, the internal carotid artery $(10,11)$. The internal carotid artery is a terminal branch of the common carotid artery. It arises from the common carotid artery, which bifurcates into the internal and external carotid artery. The internal carotid artery is vitally important as it supplies the brain. When encountering patients with LSBTs, the approach of resection of LSBTs always requires careful protection of the internal carotid artery. However, how to protect the internal carotid artery effectively remains a key problem to be solved. A series of methods can be adopted as follows. i) Be quite familiar with the anatomic structure, sign and localization prior to the operation; ii) the sufficient preoperative imaging assessment can improve the protective effect of the internal
Table IV. Complications of surgical treatments.

\begin{tabular}{lrr}
\hline & \multicolumn{2}{c}{ Total } \\
\cline { 2 - 3 } Complication & $\mathrm{N}$ & $\%$ \\
\hline Temporary paralysis of facial nerve & 5 & 24 \\
Frey's syndrome & 2 & 10 \\
Unilateral paralysis of the vocal cords & 2 & 10 \\
Bleeding & 1 & 5 \\
Dysphagia & 1 & 5 \\
Ttrismus & 1 & 5 \\
Total & 12 & 57 \\
\end{tabular}

Table V. Results of histopathological analysis.

\begin{tabular}{lrr}
\hline & \multicolumn{2}{c}{ Total } \\
\cline { 2 - 3 } Histopathology & $\mathrm{N}$ & $\%$ \\
\hline Benign tumors & 14 & 67 \\
Pleomorphic adenoma & 9 & 43 \\
Schwannoma & 3 & 14 \\
Inflammatory myofibroblastic tumor & 1 & 5 \\
Carotid body paraganglioma & 1 & 5 \\
Malignant tumors & 7 & 33 \\
Chondroblastoma & 1 & 5 \\
Maxillary sinus carcinoma & 1 & 5 \\
Osteosarcoma & 1 & 5 \\
Gingival squamous cell carcinomas & 1 & 5 \\
Myxofibrosarcoma & 1 & 5 \\
Malignant fibrous histiocytoma & 1 & 5 \\
Mucoepidermoid carcinoma & 1 & 5 \\
\hline
\end{tabular}

carotid artery; iii) be careful while opening the carotid sheath that contains the common carotid artery, the internal carotid artery, the internal jugular vein and the vagus nerve; iv) if opening the carotid sheath and exposing its contents is necessary, it is essential to mark the contents for recognition and protection; v) sufficient attention has to be paid to avoid the vital vessels and nerves when using postoperative drainage. Whichever method is adopted, care, patience and caution are absolutely required.

From our cases, the benign tumors $(66.7 \%)$ hold a significant predominance, which is the same as that in the majority of articles we reviewed $(6,12,13)$. Additionally, pleomorphic adenoma is the most common histological type. Beyond that, however, a group of rare tumors, such as chondroblastoma, fibrohistiocytoma and inflammatory myofibroblastic tumors, existed in the LSB area on account of the high complexity of body tissues and structures $(5,12,14,15)$. The majority of LSBTs derive from the parotid gland, whereas neurogenic tumors occurred less frequently in our patient series.

Consequently, preoperative imaging, especially CT and MRI, is of vital importance in terms of in choosing the best 
diagnostic and the most proper surgical approach for masses of the LSB. Usually, the CT and MRI images can both be used as the first option. MRI supplies us with more information, by and large. However, CT images are able to gain a better demarcation between normal and lesion tissues $(16,17)$. Therefore, it was the most widely used method in our cases on account of its greater level of acceptance and lower cost. In addition to these, angiography was indicated for tumors that originated in vessels, and in certain patients where embolization needed to be performed, the angiography had to be carried out 2 or 3 days before surgery (18-20). All of these radiological studies supplied us with information about the vital vessels, the location of masses and the relationships among the various tissues nearby. With the help of CT, MRI and angiography, decisions can be made by analyzing the location, size and character of masses in the LSB area.

Nowadays, it remains unclear whether the effect of fine needle aspiration biopsy (FNAB) can be taken as part of the conventional preoperative assessment of patients with LSBTs. Some researchers have reported that they took an FNAB during an evaluation of patients when the possibility of an open biopsy was precluded, and concluded that FNAB might be a useful tool in this situation (21-23). However, there remain several researchers who do not hold with this opinion. They consider that FNAB results are not accurate enough (24-26). Hence, we did not use FNAB in our cases. Furthermore, we considered that, when the diagnosis of vascular diseases is ruled out by imaging results, FNAB is more appropriate for patients with masses located in relatively superficial areas. Moreover, the preoperative diagnosis may be much more meaningful for metastatic diseases, lymphomas and other malignancies (27).

Due to the anatomical complexity and low morbidity of LSBTs, diagnosis and treatment is quite difficult for surgeons. Patients with LSBTs are willing to accept operations to remove the lesion. Although different surgical approaches have been suggested for the complete resection of lesions of the LSB, access to this complex and variable region remains somewhat difficult due to the proximity to vital neurovascular structures and the obstruction of bones. In our cases, the reason that we chose the transmandibular, transmaxillary, transparotid-transmandibular and transcervical approaches as our surgical approaches of choice depended on the tumor location, the relationship of the crucial nerves and vessels, and the dubiety of malignancy. The transmandibular approach with or without osteotomy was the most used treatment in our cases. Owing to the limited visual field of the deep part of the intratemporal fossa area and its relatively large operating distance to the LSB area, in our opinion it is suitable for LSBTs that are mainly derived from the parapharyngeal space. As a classic means of access described by Fisch and Pillsbury $(28,29)$, the transmaxillary approach provides surgeons with a great exposure for the operation. However, this approach may result in vital nerve injury by translocation and dysfunction of the he temporal mandibular joint (TMJ). In addition, the risk of facial deformity resulting from facial incision or maxilla osteotomy is another potential drawback. The transparotid-transmandibular approach, which could be considered as a variant of the transmandibular approach, was proposed by Sekhar et al (30). This approach comprises improvements on both the visual field and the operating distance, so that a wider and more direct exposure for lesions that are derived from the deep parotid lobe may be obtained. However, the restriction of the primary lesion location remains its main shortcoming. The transcervical approach exposes the LSB to an inferior access without mandible and maxillary osteotomy. As a consequence, it is able to provide a good protection of crucial neurovascular structures of facial and deep tissues. However, its limitation is that the exposure of the LSB is relatively insufficient. For instance, it may be hard to reach the LSB region in some large masses via this access. Outside of these four surgical approaches, the transmandibular-zygomatic (31), transoral (32) and combined approach (33) have been described to cope with LSB lesions, and these methods have achieved their objectives as well. We do not propose to go further into the details about them here.

The postoperative complications are listed in Table IV. However, these did not occur randomly. In our cases, temporary paralysis of the facial nerve easily occurred when patients were subjected to the transparotid-transmandibular, the transmandibular and the transmaxillary approaches. Frey's syndrome mostly occurred when surgeons adopted the transparotid-transmandibular and the transmaxillary approaches. Unilateral paralysis of the vocal cords and dysphagia occurred after operations with the transcervical approach. Bleeding and trismus occurred following surgery with the patient for whom the combined approach was applied. Especially bleeding, irrespective of what kind of approach is applied by the surgeon, following surgery is a possibility, and this is the most dangerous problem. By reason of the complexity of LSB surgeries, the postoperative complications are not rare. Hence, there is a risk of intricate problems occurring when care is not taken to reduce damage to the neurovascular structures as much as possible, and a good hemostasis intraoperatively is not achieved, also taking into consideration the corresponding postoperative treatments $(4,27)$.

All of our cases were followed up to check for recurrence after surgical operations. We found the recidivation of malignant tumors was significantly higher than benign tumors, and malignant ones always had a worse prognosis. These findings were in accordance with those of other studies $(4,6)$.

In our cases, the follow-up of our patients with malignancies of LSB revealed that postoperative radiotherapy or chemotherapy did not reach an ideal treatment outcome. There was an apparent selective bias in our small group of patients, and thus we could not conclude that radiotherapy or chemotherapy had no effect on improving the survival of patients with malignancies of LSB.

Although LSBTs have a low incidence rate and various pathologies, the majority of these tumors are treatable. Owing to the unique anatomical structure of the LSB, masses tend to be symptomless at the early stages. Therefore, clinicians should be aware of the possibility of the occurrence of LSBTs when patients present with a facial mass, medial displacement or enlargement of the pharyngeal wall. In general, radiology, CT and MRI are the major methods of diagnosis. However, in certain cases, conventional angiography or FNAB are required to confirm the diagnosis.

The majority of patients receive surgery for the removal of the lesions. Successful surgery should achieve total tumor 
resection with minimum sequelae, although this depends on the location of the tumor, as well as the structures involved. By contrast, the transparotid-transmandibular approach is suitable for the majority of LSBTs derived from the deep parotid lobe, or it requires a vast surgical field to ensure clean margins. The transcervical approach is more appropriate for LSBTs that stem from the poststyloid parapharyngeal space subdivision with benign characteristics, while it is crucial to identify and protect the vital vessels and nerves of the neck. Complex cases for which a single approach would not be sufficient may require the combined approach.

\section{References}

1. Hazarika P, Sahota JS, George S and Raja A: Surgical treament of lateral skull base tumours our experience. Indian J Otolaryngol Head Neck Surg 2: 19-22, 1993.

2. Pai PS, Moiyadi A and Nair D: Management of lateral skull base tumours. Indian J Surg Oncol 1: 125-132, 2010.

3. Kuet ML, Kasbekar AV, Masterson L and Jani P: Management of tumors arising from the parapharyngeal space: A systematic review of 1,293 cases reported over 25 years. Laryngoscope 125: 1372-1381, 2015.

4. Riffat F, Dwivedi RC, Palme C, Fish B and Jani P: A systematic review of 1143 parapharyngeal space tumors reported over 20 years. Oral Oncol 50: 421-430, 2014

5. Spinazzi EF, Desai SV, Fang CH, Jyung RW, Liu JK, Baredes S and Eloy JA: Lateral skull base Inflammatory pseudotumor: A systematic review. Laryngoscope 125: 2593-2600, 2015.

6. Khafif A, Segev Y, Kaplan DM, Gil Z and Fliss DM: Surgical management of parapharyngeal space tumors: A 10-year review. Otolaryngol Head Neck Surg 132: 401-406, 2005.

7. Jian-feng L, Qiu-hang Z, Da-zhang Y and Qiu-yi Q: Transcervical approach for resection of lateral skull base tumors. J Otology 2: 102-108, 2007.

8. Prasad SC, Piccirillo E, Chovanec M, La Melia C, De Donato G and Sanna M: Lateral skull base approaches in the management of benign parapharyngeal space tumors. Auris Nasus Larynx 42 189-198, 2015.

9. Dimitrijevic MV, Jesic SD, Mikic AA, Arsovic NA and Tomanovic NR: Parapharyngeal space tumors: 61 case reviews. Int J Oral Maxillofac Surg 39: 983-989, 2010.

10. Jacquesson T, Simon E, Berhouma M and Jouanneau E: Anatomic comparison of anterior petrosectomy versus the expanded endoscopic endonasal approach: Interest in petroclival tumors surgery. Surg Radiol Anat 37: 1199-1207, 2015.

11. Kirsch CF: Advances in magnetic resonance imaging of the skull base. Int Arch Otorhinolaryngol 18 (Suppl 2): S127-S135, 2014.

12. Rong BG, Chen WL, Ding YP, Xie G, Chen Y and Wang TD: Surgical approaches to the skull base neoplasms. Zhonghua Er Bi Yan Hou Tou Jing Wai Ke Za Zhi 40: 291-294, 2005 (In Chinese).

13. Fernández Ferro M, Fernández Sanromán J, Costas López A, Sandoval Gutiérrez J and López de Sánchez A: Surgical treatment of benign parapharyngeal space tumours. Presentation of two clinical cases and revision of the literature. Med Oral Patol Oral Cir Bucal 13: E61-E64, 2008.

14. Reid LB, Wong DS and Lyons B: Chondroblastoma of the temporal bone: A case series, review, and suggested management strategy. Skull Base Rep 1: 71-82, 2011.

15. Maire JP, Eimer S, San Galli F, Franco-Vidal V, Galland-Girodet S, Huchet A and Darrouzet V: Inflammatory myofibroblastic tumour of the skull base. Case Rep Otolaryngol 2013: 103646, 2013.
16. Carrau RL, Weissman JL, Janecka IP, Snyderman CH, Curtin HD, Sekhar L and Lee HS: Computerized tomography and magnetic resonance imaging following cranial base surgery. Laryngoscope 101: 951-959, 1991.

17. Jiang ZY, Allen K, KutZ JW Jr and Isaacson B: Clinical impact of early CT scans after lateral skull-base surgery. Otolaryngol Head Neck Surg 149: 786-788, 2013.

18. Durden DD and Williams DW III: Radiology of skull base neoplasms. Otolaryngol Clin North Am 34: 1043-1064.vii, 2001.

19. Meli GA, Chiaramonte R, Cavallaro T, Puglisi C and Pero G: Carotid body paraganglioma. Diagnosis and treatment by angiography. Neuroradiol J 19: 645-648, 2006.

20. Tong Y: Role of duplex ultrasound in the diagnosis and assessment of carotid body tumour: A literature review. Intractable Rare Dis Res 1: 129-133, 2012.

21. Farrag TY, Lin FR, Koch WM, Califano JA, Cummings CW, Farinola MA and Tufano RP: The role of pre-operative CT-guided FNAB for parapharyngeal space tumors. Otolaryngol Head Neck Surg 136: 411-414, 2007

22. Papadogeorgakis N, Petsinis V, Goutzanis L, Kostakis G and Alexandridis C: Parapharyngeal space tumors: Surgical approaches in a series of 13 cases. Int J Oral Maxillofac Surg 39: 243-250, 2010.

23. Cramer $\mathrm{H}$, Lampe $\mathrm{H}$ and Downing P: Intraoral and transoral fine needle aspiration. A review of 25 cases. Acta Cytol 39: 683-688, 1995.

24. Suárez-Fente V, Llorente-Pendás JL, Gómez-Martínez J, García-González LA, López-Álvarez F and Suárez-Nieto C: Primary tumours of the parapharyngeal space. Our experience in 51 patients. Acta Otorrinolaringol Esp 60: 19-24, 2009 (In Spanish).

25. Luna-Ortiz K, Navarrete-Alemán JE, Granados-Garcia M and Herrera-Gómez A: Primary parapharyngeal space tumors in a Mexican cancer center. Otolaryngol Head Neck Surg 132: 587-591, 2005.

26. Hughes KV III, Olsen KD and McCaffrey TV: Parapharyngeal space neoplasms. Head Neck 17: 124-130, 1995.

27. Cassoni A, Terenzi V, Della Monaca M, Bartoli D, Battisti A, Rajabtork Zadeh O and Valentini V: Parapharyngeal space benign tumours: Our experience. J Craniomaxillofac Surg 42: 101-105, 2014.

28. Fisch U: Infratemporal fossa approach to tumours of the temporal bone and base of the skull. J Laryngol Otol 92: 949-967, 1978

29. Fisch U and Pillsbury HC: Infratemporal fossa approach to lesions in the temporal bone and base of the skull. Arch Otolaryngol 105: 99-107, 1979.

30. Sekhar LN, Schramm VL Jr and Jones NF: Subtemporalpreauricular infratemporal fossa approach to large lateral and posterior cranial base neoplasms. J Neurosurg 67: 488-499, 1987.

31. Donovan MG, Ondra SL, Illig JJ and Dickerson NC: Combined transmandibular-zygomatic approach and infratemporal craniotomy for intracranial skull base tumors. J Oral Maxillofac Surg 51: 754-758, 1993.

32. Carrau RL, Myers EN and Johnson JT: Management of tumors arising in the parapharyngeal space. Laryngoscope 100: 583-589, 1990.

33. Betka J, Chovanec M, Klozar J, Taudy M, Plzák J, Kodetová D and Lisý J: Transoral and combined transoral-transcervical approach in the surgery of parapharyngeal tumors. Eur Arch Otorhinolaryngol 267: 765-772, 2010. 